Homology, Homotopy and Applications, vol.6(1), 2004, pp.167-173

\title{
HOMOTOPY LIE ALGEBRA OF CLASSIFYING SPACES FOR HYPERBOLIC COFORMAL 2-CONES
}

\author{
J.-B. GATSINZI \\ (communicated by Johannes Huebschmann)
}

\section{Abstract}

In this paper, we show that the rational homotopy Lie algebra of classifying spaces for certain types of hyperbolic coformal 2 -cones is not nilpotent.

\section{Introduction}

A simply connected space $X$ is called an $n$-cone if it is built up by a sequence of cofibrations

$$
Y_{k} \stackrel{f}{\rightarrow} X_{k-1} \stackrel{j_{k}}{\rightarrow} X_{k}
$$

with $X_{0}=*$ and $X_{n} \simeq X$. One can further assume that $Y_{k} \simeq \Sigma^{k-1} W_{k}$ is a $(k-1)$ fold suspension of a connected space $W_{k}$ [3]. In particular a 2-cone $X$ is the cofibre of a map between two suspensions

$$
\Sigma A \stackrel{f}{\rightarrow} \Sigma B \rightarrow X .
$$

Spaces under consideration are assumed to be 1-connected and of finite type, that is, $H^{i}(X ; \mathbb{Q})$ is a finite-dimensional $\mathbb{Q}$-vector space. To every space $X$ corresponds a free chain Lie algebra of the form $(\mathbb{L}(V), \delta)[\mathbf{2}]$, called a Quillen model of $X$. It is an algebraic model of the rational homotopy type of $X$. In particular, one has an isomorphism of Lie algebras $H_{*}(\mathbb{L}(V), \delta) \cong \pi_{*}(\Omega X) \otimes \mathbb{Q}$. The model is called minimal if $\delta V \subset \mathbb{L} \geqslant 2(V)$. A space $X$ is called coformal if there is a map of differential Lie algebras $(\mathbb{L}(V), \delta) \rightarrow\left(\pi_{*}(\Omega X) \otimes \mathbb{Q}, 0\right)$ that induces an isomorphism in homology. Any continuous map $f: X \rightarrow Y$ has a Lie representative $\tilde{f}:\left(\mathbb{L}(W), \delta^{\prime}\right) \rightarrow(\mathbb{L}(V), \delta)$ between respective models of $X$ and $Y$.

If $X$ is a 2-cone as defined by (1) and $\tilde{f}: \mathbb{L}(W) \rightarrow \mathbb{L}(V)$ is a model of $f$, then a Quillen model of the cofibre $X$ of $f$ is obtained as the push out of the following diagram:

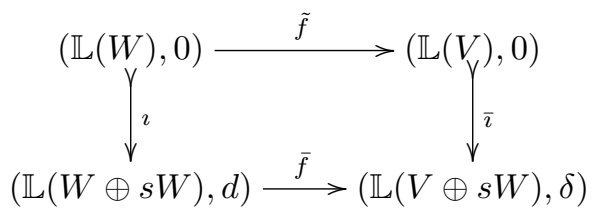

This work was supported by the Abdus Salam ICTP in cooperation with SIDA. Received November 14, 2003, revised April 21, 2004; published on May 3, 2004. 2000 Mathematics Subject Classification: Primary 55P62; Secondary 55M30.

Key words and phrases: rational homotopy, coformal spaces, 2-cones, differential Ext. (C) 2004, J.-B. Gatsinzi. Permission to copy for private use granted. 
where $(\mathbb{L}(W \oplus s W), d)$ is acyclic. Moreover the differential on $\mathbb{L}(V \oplus s W)$ verifies $\delta s W \subset \mathbb{L}(V)$. Hence a 2-cone $X$ has a Quillen model of the form $\left(\mathbb{L}\left(V_{1} \oplus V_{2}\right), \delta\right)$ such that $\delta V_{1}=0$ and $\delta V_{2} \subset \mathbb{L}\left(V_{1}\right)$.

A Sullivan model of a space $X$ is a cochain algebra $(\wedge Z, d)$ that algebraically models the rational homotopy type of $X$. In particular, one has an isomorphism of graded algebras $H^{*}(\wedge Z, d) \cong H^{*}(X ; \mathbb{Q})$. The model is called minimal if $d Z \subset \wedge^{\geqslant 2} Z$. In this case the vector spaces $Z^{n}$ and $\operatorname{Hom}\left(\pi_{n}(X), \mathbb{Q}\right)$ are isomorphic. If $X$ has the rational homotopy type of a finite $\mathrm{CW}$-complex, we say that $X$ is elliptic if $Z$ is finite dimensional, otherwise $X$ is called hyperbolic.

\section{Models of classifying spaces}

Henceforth $X$ will denote a simply connected finite CW-complex and $\mathcal{L}_{X}$ its homotopy Lie algebra. Let aut $X$ denote the space of free self homotopy equivalences of $X$, aut $_{1}(X)$ the path component of aut $X$ containing the identity map of $X$. The space $\operatorname{Baut}_{1}(X)$ classifies fibrations with fibre $X$ over simply connected base spaces [4].

The Schlessinger-Stasheff model for $\operatorname{Baut}_{1}(X)$ is defined as follows [12]. If $(\mathbb{L}(V), \delta)$ is a Quillen model of $X$, we define a differential Lie algebra $\operatorname{Der} \mathbb{L}(V)=$ $\oplus_{k} \geqslant 1 \operatorname{Der}_{k} \mathbb{L}(V)$ where $\operatorname{Der}_{k} \mathbb{L}(V)$ is the vector space of derivations of $\mathbb{L}(V)$ which increase the degree by $k$, with the restriction that $\operatorname{Der}_{1} \mathbb{L}(V)$ is the vector space of derivations of degree 1 that commute with the differential $\delta$.

Define the differential Lie algebra $(s \mathbb{L}(V) \oplus \operatorname{Der} \mathbb{L}(V), D)$ as follows:

- The graded vector space $s \mathbb{L}(V) \oplus \operatorname{Der} \mathbb{L}(V)$ is isomorphic to $s \mathbb{L}(V) \oplus \operatorname{Der} \mathbb{L}(V)$,

- If $\theta, \gamma \in \operatorname{Der} \mathbb{L}(V)$ and $s x, s y \in s \mathbb{L}(V)$, then $[\theta, \gamma]=\theta \gamma-(-1)^{|\theta||\gamma|} \gamma \theta,[\theta, s x]=$ $(-1)^{|\theta|} s \theta(x)$ and $[s x, s y]=0$,

- The differential $D$ is defined by $D \theta=[\delta, \theta], D(s x)=-s \delta x+a d x$, where $a d x$ is the inner derivation determined by $x$.

From the Sullivan minimal model $(\wedge Z, d)$, Sullivan defines the graded differential Lie algebra $(\operatorname{Der} \wedge Z, D)$ as follows $\left[\mathbf{1 3}\right.$. For $k>1$, the vector space $(\operatorname{Der} \wedge Z)_{k}$ consists of the derivations on $\wedge Z$ that decrease the degree by $k$ and $(\operatorname{Der} \wedge Z)_{1}$ is the vector space of derivations of degree 1 verifying $d \theta+\theta d=0$. For $\theta, \gamma \in \operatorname{Der} \wedge V$, the Lie bracket is defined by $[\theta, \gamma]=\theta \gamma-(-1)^{|\theta||\gamma|} \gamma \theta$ and the differential $D$ is defined by $D \theta=[d, \theta]$.

We have the following result:

Theorem 1. [13, 12, 14] The differential Lie algebras $(\operatorname{Der} \wedge Z, D)$ and $(s \mathbb{L}(V) \oplus \operatorname{Der} \mathbb{L}(V), D)$ are models of the classifying space $B$ aut $t_{1}(X)$.

An indirect proof of the Schlessinger-Stasheff model is given in [8, Theorem 2].

\section{The classifying space spectral sequence}

Recall that if $(L, \delta)$ is a graded differential Lie algebra, then $L$ becomes an $U L$ module by the adjoint representation $a d: L \rightarrow \operatorname{Hom}(L, L)$. In the sequel all Lie 
algebras are endowed with the above module structure.

Let $(\mathbb{L}(V), \delta)$ be a Quillen model of a finite $\mathrm{CW}$-complex and $(T V, d)$ its enveloping algebra. On the $T V$-module $T V \otimes(\mathbb{Q} \oplus s V)$, define a $\mathbb{Q}$-linear map

$$
S: T V \otimes(\mathbb{Q} \oplus s V) \rightarrow T V \otimes(\mathbb{Q} \oplus s V)
$$

as follows:

- $S(1 \otimes x)=0$ for all $x \in \mathbb{Q} \oplus s V$,

- $S(v \otimes 1)=1 \otimes s v$ for all $v \in V$,

- If $a \in T V$ and $x \in T V \otimes(\mathbb{Q} \oplus s V)$ with $|x|>0$, then $S(a \cdot x)=(-1)^{|a|} a \cdot S(x)$.

The differential on the $T V$-module $T V \otimes(\mathbb{Q} \oplus s V)$ is defined by

$$
D(1 \otimes s v)=v \otimes 1-S(d v \otimes 1) \text { for } v \in V \text { and } D(1 \otimes 1)=0 .
$$

It follows from $[\mathbf{1}]$ that $(T V \otimes(\mathbb{Q} \oplus s V), D)$ is acyclic, hence it is a semifree resolution of $\mathbb{Q}$ as a $(T V, d)$-module $[\mathbf{6}, \S 6]$.

Using the Schlessinger-Stasheff model of the classifying space, the author proved the following:

Theorem 2. [8] The differential graded vector spaces $H_{\text {or }}{ }_{T V}(T V \otimes(\mathbb{Q} \oplus s V), \mathbb{L}(V))$ and $s \mathbb{L}(V) \oplus \operatorname{Der} \mathbb{L}(V)$ are isomorphic. Moreover, for $n \geqslant 0$, the $\mathbb{Q}$-vector spaces $\operatorname{Ext}_{T V}^{n}(\mathbb{Q}, \tilde{L}(V))$ and $\pi_{n+1}\left(\Omega B\right.$ aut $\left.t_{1} X\right) \otimes \mathbb{Q}$ are isomorphic.

In particular if $X$ is a coformal space, one has an isomorphism $\pi_{n}\left(B\right.$ aut $\left.t_{1} X\right) \otimes \mathbb{Q} \cong$ $\operatorname{Ext}_{U \mathcal{L}_{X}}^{n}\left(\mathbb{Q}, \mathcal{L}_{X}\right)$. Therefore $\pi_{*}\left(B\right.$ aut $\left._{1} X\right) \otimes \mathbb{Q}$ can be computed by the means of a projective resolution of $\mathbb{Q}$ as an $U \mathcal{L}_{X}$-module.

Consider the complex $\mathcal{P}=H_{T} m_{T V}(T V \otimes(\mathbb{Q} \oplus s V), \mathbb{L}(V))$. Filter $V$ as follows

$$
F_{0} V=0, \quad F_{p+1} V=\left\{x \in V: d x \in \mathbb{L}\left(F_{p} V\right)\right\} .
$$

We will denote $V_{p}=F_{p} V / F_{p-1} V$. If $F_{n-1} V \neq F_{n} V=V$, following Lemaire [10] we say that $V$ is of length $n$. We will restrict to spaces with a Quillen model of length $n$.

Define a filtration on $P=T V \otimes(\mathbb{Q} \oplus s V)$ as follows:

$$
P_{0}=T V \otimes \mathbb{Q}, P_{1}=T V \otimes\left(\mathbb{Q} \oplus s V_{1}\right), \ldots, P_{n}=T V \otimes\left(\mathbb{Q} \oplus s V_{\leqslant n}\right) .
$$

We filter the complex

$$
\operatorname{Hom}_{T V}(T V \otimes(\mathbb{Q} \oplus s V), \mathbb{L}(V))
$$

by

$$
F_{k}=\left\{f: f\left(P_{k-1}\right)=0\right\} .
$$

This yields a spectral sequence $E_{r}$ such that $E_{1}^{p, q}=\operatorname{Hom}_{\mathbb{Q}}\left(s V_{p}, \mathcal{L}_{X}\right)$ for $p>1$, $E_{1}^{0, q}=\operatorname{Hom}_{\mathbb{Q}}\left(\mathbb{Q}, \mathcal{L}_{X}\right)$ and that converges to $\operatorname{Ext}_{T V}^{*}(\mathbb{Q}, \mathbb{L}(V))$. This sequence will be called the classifying space spectral sequence of $X$.

Now assume that $X$ is coformal and let $A=U \mathcal{L}_{X}$. If $\mathbb{L}\left(V_{1}\right) / I$ is a minimal presentation of $\mathcal{L}_{X}$, then there is a quasi-isomorphism $\left(\mathbb{L}\left(V_{1} \oplus V_{2} \oplus \cdots \oplus V_{n}\right), \delta\right) \rightarrow \mathcal{L}_{X}$ which extends to $p:(T V, d) \stackrel{\simeq}{\longrightarrow}(A, 0)$. The $\left(E_{1}, d\right)$ term provides a resolution

$$
\cdots \rightarrow A \otimes s V_{n} \rightarrow A \otimes s V_{n-1} \rightarrow \cdots \rightarrow A \otimes s V_{1} \rightarrow A \rightarrow \mathbb{Q}
$$


of $\mathbb{Q}$ as an $A$-module. Here the differential is given by the composition

$$
s V_{n} \stackrel{D}{\longrightarrow} T V \otimes\left(\mathbb{Q} \oplus s V_{n-1}\right) \stackrel{p \otimes i d}{\longrightarrow} A \otimes\left(\mathbb{Q} \oplus s V_{n-1}\right) .
$$

The spectral sequence will therefore collapse at $E_{2}$ level. Moreover $\operatorname{Ext}_{A}^{*}\left(\mathbb{Q}, \mathcal{L}_{X}\right)$ is endowed with a Lie algebra structure verifying

$$
\left[\mathrm{Ext}^{p, *}, \mathrm{Ext}^{q, *}\right] \subset \operatorname{Ext}^{p+q-1, *} .
$$

The Lie bracket can be defined using the bijection between the Koszul complex $C^{*}\left(\mathcal{L}_{X}, \mathcal{L}_{X}\right)$ and derivations on the Sullivan model $C^{*}\left(\mathcal{L}_{X}, \mathbb{Q}\right)$ of $X[\mathbf{9}$, Proposition 4] (see also [7] for a direct definition of the Lie bracket on $C^{*}\left(\mathcal{L}_{X}, \mathcal{L}_{X}\right)$ ). Alternatively one may use the bijection

$$
\operatorname{Hom}_{T V}(T V \otimes(\mathbb{Q} \oplus s V), \mathbb{L}(V)) \cong s \mathbb{L}(V) \oplus \operatorname{Der} \mathbb{L}(V)
$$

to transfer a Lie algebra structure on $\operatorname{Hom}_{T V}(T V \otimes(\mathbb{Q} \oplus s V), \mathbb{L}(V))$ from

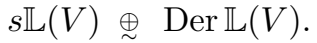

Definition 3. Let $L$ be a Lie algebra. An element $x \in L$ is called locally nilpotent if for every $y \in L$, there is a positive integer $k$ such that $(\operatorname{ad} x)^{k}(y)=0$. A subset $K \subset L$ is called locally nilpotent if each element of $K$ is locally nilpotent.

We deduce from Equation (2) the following

Proposition 4. Let $X$ be a coformal space of homotopy Lie algebra denoted $\mathcal{L}_{X}$. If $X$ has a Quillen model $(\mathbb{L}(V), \delta)$, of length $n$, one has:

1. For $k \neq 1, \operatorname{Ext}_{A}^{k}\left(\mathbb{Q}, \mathcal{L}_{X}\right)$ is locally nilpotent,

2. $\operatorname{Ext}_{A}^{1}\left(\mathbb{Q}, \mathcal{L}_{X}\right)$ is a subalgebra of $\operatorname{Ext}_{A}\left(\mathbb{Q}, \mathcal{L}_{X}\right)$,

3. If $\operatorname{Ext}_{A}^{0}\left(\mathbb{Q}, \mathcal{L}_{X}\right)=0$, then $\oplus_{i \geqslant i_{0}} \operatorname{Ext}_{A}^{i}\left(\mathbb{Q}, \mathcal{L}_{X}\right)$ is an ideal of $\operatorname{Ext}_{A}\left(\mathbb{Q}, \mathcal{L}_{X}\right)$, for $i_{0} \geqslant 1$.

We will now assume that $X$ is a coformal 2-cone. Recall that $X$ has a Quillen minimal model of the form $\left(\mathbb{L}\left(V_{1} \oplus V_{2}\right), \delta\right)$, with $\delta V_{1}=0$ and $\delta V_{2} \subset \mathbb{L}\left(V_{1}\right)$. Moreover $\pi_{*}(\Omega X) \otimes \mathbb{Q}=H_{*}\left(\mathbb{L}\left(V_{1} \oplus V_{2}\right), \delta\right)=\mathbb{L}\left(V_{1}\right) / I$, where $I$ is the ideal of $\mathbb{L}\left(V_{1}\right)$ generated by $\delta V_{2}$.

Definition 5. Let $\mathbb{L}(V)$ be a free Lie algebra where $\{a, b, c, \ldots\}$ is a basis of $V$. Denote $\mathbb{L}^{n}(V)$ the subspace of $\mathbb{L}(V)$ consisting of Lie brackets of length $n$. Consider a basis $\left\{u_{1}, u_{2}, \ldots\right\}$ of $\mathbb{L}^{n}(V)$ where each $u_{i}$ is a Lie monomial. If $x \in\{a, b, c, \ldots\}$, we define the length of $u_{i}$ in the variable $x, l_{x}\left(u_{i}\right)$, as the number of occurrences of the letter $x$ in $u_{i}$. If $u=\sum r_{i} u_{i} \in \mathbb{L}^{n}(V)$, define $l_{x}(u)=\min \left\{l_{x}\left(u_{i}\right)\right\}$ and if $v=\sum v_{i}$ where $v_{i} \in \mathbb{L}^{i}(V), l_{x}(v)=\min \left\{l_{x}\left(v_{i}\right)\right\}$.

It is straightforward that the above definition extends to the enveloping algebra $T(V)$.

Theorem 6. Let $X$ be a coformal 2-cone and $\left(\mathbb{L}\left(V_{1} \oplus V_{2}\right), \delta\right)$ be its Quillen minimal model. Choose a basis $\left\{x_{1}, x_{2}, \ldots\right\}$ for $V_{1}$ and a basis $\left\{y_{1}, y_{2}, \ldots\right\}$ for $V_{2}$. If for some $x_{k} \in\left\{x_{1}, x_{2}, \ldots\right\}, l_{x_{k}}\left(\delta y_{j}\right) \geqslant 2$ for all $y_{j} \in\left\{y_{1}, y_{2}, \ldots\right\}$, then $\operatorname{Ext}_{A}^{2, *}\left(\mathbb{Q}, \mathcal{L}_{X}\right)$ is infinite dimensional. 
Proof. Note that for $i \neq k$ the element $\left(a d x_{i}\right)^{n}\left(x_{k}\right)$ is a nonzero homology class in $H_{*}\left(\mathbb{L}\left(V_{1} \oplus V_{2}\right), \delta\right)$ as it contains only one occurrence of $x_{k}$. Take $y_{t} \in\left\{y_{1}, y_{2}, \ldots\right\}$ and $x_{m} \in\left\{x_{1}, x_{2}, \ldots\right\}$ with $m \neq k$. For each $n \geqslant 1$, define $f_{n} \in \operatorname{Hom}_{A}(A \otimes$ $\left.s V_{2}, \mathcal{L}_{X}\right)$ by $f_{n}\left(s y_{t}\right)=\left(a d x_{m}\right)^{n}\left(x_{k}\right)$ and $f_{n}\left(s y_{j}\right)=0$ for $j \neq t$. Obviously $f_{n} \in$ $\operatorname{Hom}_{A}\left(A \otimes s V_{2}, \mathcal{L}_{X}\right)$ is a cocycle. Suppose that $f_{n}$ is a coboundary. There exists $g_{n} \in \operatorname{Hom}_{A}\left(A \otimes s V_{1}, \mathcal{L}_{X}\right)$ such that $f_{n}\left(s y_{t}\right)=g_{n}\left(d s y_{t}\right)$. From the definition of the differential $d$, one has $d s y_{t}=\sum_{i} p_{i} s x_{i}$, where the $p_{i}$ 's are polynomials in the variables $x_{1}, x_{2}, \ldots$. From the hypothesis on the differential $d y_{t}$ one knows that $l_{x_{k}}\left(p_{i}\right) \geqslant 2$ for $i \neq k$ and $l_{x_{k}}\left(p_{k}\right) \geqslant 1$. By using the number of occurrences of the variable $x_{k}$, one deduces from the previous equalities that $\left(a d x_{m}\right)^{n}\left(x_{k}\right)$ equals the component of length 1 in $x_{k}$ of $p_{k} g_{n}\left(s x_{k}\right)$. Therefore, in the monomial decomposition of $g_{n}\left(s x_{k}\right)$ (resp. $\left.p_{k}\right)$ there must exist $\left(a d x_{m}\right)^{n-s}\left(x_{k}\right)$ (resp. $x_{m}^{s}$ ). We obtain a contradiction with $l_{x_{k}}\left(p_{k}\right) \geqslant 1$.

The cocycles $f_{n}$ create an infinite number of non zero classes (of distinct degrees) and the space $\operatorname{Ext}_{A}^{2, *}\left(\mathbb{Q}, \mathcal{L}_{X}\right)$ is infinite dimensional.

Corollary 7. If hypotheses of the above theorem are satisfied, then $\operatorname{cat}\left(B\right.$ aut $\left.t_{1}(X)\right)=$ $\infty$.

Proof. If $s x \in \mathrm{Ext}^{0, *} \subset \mathbb{L}\left(V_{1}\right) / I$ and $f \in \mathrm{Ext}^{2, *}$ then $[f, s x]= \pm s f(x)$. As elements of $\mathrm{Ext}^{2, *}$ vanish on $V_{1}$, we deduce that $\left[\mathrm{Ext}^{2, *}, \mathrm{Ext}^{0, *}\right]=0$. It follows from Theorem 6 that $J=\operatorname{Ext}_{U \mathcal{L}_{X}}^{2}\left(\mathbb{Q}, \mathcal{L}_{X}\right)$ is an infinite dimensional ideal of $\pi_{*}\left(\Omega B\right.$ aut $\left.t_{1}(X)\right)$. Moreover it follows from Equation (2) that $J$ is abelian. We deduce that the category of $B$ aut $_{1}(X)$ is infinite [5, Theorem 12.2].

If $X$ is an elliptic space of Sullivan minimal model $(\wedge Z, d)$ then $\operatorname{Der} \wedge Z$ is a finite dimensional $\mathbb{Q}$-vector space. Hence the homotopy Lie algebra of $B$ aut $t_{1}(X)$ is finite dimensional, therefore $\pi_{*}\left(\Omega B\right.$ aut $\left.{ }_{1}(X)\right) \otimes \mathbb{Q}$ is nilpotent. In [11], P. Salvatore proved that if $X=S^{2 n+1} \vee S^{2 n+1}$, then $\pi_{*}\left(\Omega B\right.$ aut $\left.t_{1}(X)\right) \otimes \mathbb{Q}$ contains an element $\alpha$ that is not locally nilpotent. The proof consists in the construction of two outer derivations $\alpha$ and $\beta$ of the free Lie algebra $\mathbb{L}(a, b)$, where $|a|=|b|=2 n$, such that $(a d \alpha)^{i}(\beta) \neq 0$, for every integer $i>0$. The technique can be applied to any free Lie algebra with at least two generators. Therefore $\pi_{*}(\Omega B$ aut $1(X)) \otimes \mathbb{Q}$ contains an element $\alpha$ that is not locally nilpotent if $X$ is a wedge of two spheres or more.

P. Salvatore asked if $\pi_{*}(\Omega B$ aut $1(X)) \otimes \mathbb{Q}$ has always such a property for every hyperbolic space $X$. A positive answer to this question would provide another characterization of the elliptic-hyperbolic dichotomy [5].

For a product space we have the following

Proposition 8. If $X=Y \times Z$ is a product space such that the Lie algebra $\pi_{*}\left(\Omega B\right.$ aut $\left._{1}(Y)\right) \otimes \mathbb{Q}$ is not nilpotent, then $\pi_{*}\left(\Omega B\right.$ aut $\left.t_{1}(X)\right) \otimes \mathbb{Q}$ is not nilpotent.

Proof. Let $(\wedge V, d)$ and $\left(\wedge W, d^{\prime}\right)$ be Sullivan models of $Y$ and $Z$ respectively. Therefore $\left(\wedge V \otimes \wedge W, d \otimes d^{\prime}\right)$ is a Sullivan model of $X$. It follows from [12] that

$$
H_{*}(\operatorname{Der}(\wedge V \otimes \wedge W)) \cong H_{*}(\operatorname{Der} \wedge V) \otimes H^{*}(\wedge W) \oplus H^{*}(\wedge V) \otimes H_{*}(\operatorname{Der} \wedge W) .
$$

Therefore $\pi_{*}\left(\Omega B\right.$ aut $\left._{1}(Y)\right) \otimes \mathbb{Q}$ is a subalgebra of $\pi_{*}\left(\Omega B\right.$ aut $\left.t_{1}(X)\right) \otimes \mathbb{Q}$. 
In particular if $Y$ is a wedge of at least two spheres, then the Lie algebra $\pi_{*}\left(\Omega B\right.$ aut $\left._{1}(Y)\right) \otimes \mathbb{Q}$ is not nilpotent and so is $\pi_{*}\left(\Omega B\right.$ aut $\left._{1}(X)\right) \otimes \mathbb{Q}$.

We can extend Salvatore's result to some certain types of coformal hyperbolic 2-cones.

Theorem 9. Under the hypotheses of Theorem 6, the rational homotopy Lie algebra of $B$ aut $(X)$ is not nilpotent.

Proof. For $i \neq k$, let $\left(a d x_{k}\right)^{n}\left(x_{i}\right)$ be a nonzero element of $\mathcal{L}_{X}$. Define $\alpha_{n} \in$ $\operatorname{Ext}_{A}^{1}\left(\mathbb{Q}, \mathcal{L}_{X}\right)$ by $\alpha_{n}\left(s x_{i}\right)=\left(\operatorname{ad} x_{k}\right)^{n}\left(x_{i}\right)$ and zero on the other generators of $\mathcal{L}_{X}$. Take $w \in V_{2}$ and define $\beta_{m} \in \operatorname{Ext}_{A}^{2}\left(\mathbb{Q}, \mathcal{L}_{X}\right)$ by $\beta_{m}(s w)=\left(a d x_{k}\right)^{m}\left(x_{i}\right)$ and zero elsewhere. A short computation shows that $\left[\alpha_{n}, \beta_{m}\right]= \pm \beta_{m+n}$. Hence $\left(\operatorname{ad} \alpha_{n}\right)^{l}\left(\beta_{m}\right) \neq 0$ for all $l \geqslant 1$. Therefore $\pi_{*}\left(\Omega B\right.$ aut $\left.t_{1}(X)\right) \otimes \mathbb{Q}$ is not nilpotent.

Example 10. Consider the space $X$ for which the Quillen minimal model is $(\mathbb{L}(a, b, c), d)$ with $d a=d b=0$ and $d c=[b,[b, a]]$. The space $X$ satisfies the hypothesis of Theorem 6 . Therefore $\operatorname{cat}\left(B\right.$ aut $\left._{1}(X)\right)$ is infinite. Moreover the homotopy Lie algebra of $B$ aut $_{1}(X) \otimes \mathbb{Q}$ is not nilpotent.

\section{References}

[1] J.F. Adams and P. Hilton, On the chain algebra of loop spaces, Comment. Math. Helv. 30 (1956), 305-330.

[2] H.J. Baues and J.-M. Lemaire, Minimal models in homotopy theory, Math. Ann. 225 (1977), 219-242.

[3] O. Cornea, Strong LS category equals cone-length, Topology 34 (1995), 377381.

[4] E. Dror and A. Zabrodsky, Unipotency and nilpotency in homotopy equivalences, Topology 18 (1979), 187-197.

[5] Y. Félix, La Dichotomie Elliptique Hyperbolique en Homotopie Rationnelle, Astérisque 176, 1989.

[6] Y. Félix, S. Halperin and J.-C. Thomas, Rational Homotopy Theory, Graduate Texts in Mathematics, Springer, New York, 2000.

[7] D.B. Fuchs, Cohomology of Infinite Dimensional Lie Algebras, Consultants Bureau, New York, 1986.

[8] J.-B. Gatsinzi, The homotopy Lie algebra of classifying spaces, J. Pure Appl. Algebra 120 (1997), 281-289.

[9] J.-B. Gatsinzi, Products and Lusternik-Schnirelmann category of classifying spaces, Indag. Mathem. 9 (1998), 351-357.

[10] J.-M. Lemaire, Algèbres connexes et homologie des espaces de lacets, Lecture Notes in Math., vol. 422, Springer Verlag, Berlin, 1974.

[11] P. Salvatore, Rational homotopy nilpotency of self-equivalences, Topology Appl. 77 (1997), 37-50. 
[12] M. Schlessinger and J. Stasheff, Deformations theory and rational homotopy type, preprint, 1982.

[13] D. Sullivan, Infinitesimal computations in topology, Publ. I.H.E.S. 47 (1977), 269-331.

[14] D. Tanré, Homotopie Rationnelle: Modèles de Chen, Quillen, Sullivan, Lecture Notes in Math., vol. 1025, Springer Verlag, Berlin, 1983.

This article may be accessed via WWW at http://www.rmi.acnet.ge/hha/ or by anonymous ftp at

ftp://ftp.rmi.acnet.ge/pub/hha/volumes/2004/n1a11/v6n1a11.(dvi,ps,pdf)

J.-B. Gatsinzi gatsinzj@mopipi.ub.bw

Department of Mathematics,

University of Botswana,

Private Bag 0022, Gaborone,

Botswana 